\title{
Interactions Between Forms of Memory: When Priming Hinders New Episodic Learning
}

\section{Citation}

Wagner, Anthony D., Anat Maril, and Daniel L. Schacter. 2000. Interactions between forms of memory: When priming hinders new episodic learning. Journal of Cognitive Neuroscience 12 Suppl. no. 2: 52-60.

\section{Published Version}

doi:10.1162/089892900564064

\section{Permanent link}

http://nrs.harvard.edu/urn-3:HUL.InstRepos:3606233

\section{Terms of Use}

This article was downloaded from Harvard University's DASH repository, and is made available under the terms and conditions applicable to Other Posted Material, as set forth at http:// nrs.harvard.edu/urn-3:HUL.InstRepos:dash.current.terms-of-use\#LAA

\section{Share Your Story}

The Harvard community has made this article openly available.

Please share how this access benefits you. Submit a story.

\section{Accessibility}




\title{
Interactions Between Forms of Memory: When Priming Hinders New Episodic Learning
}

\author{
Anthony D. Wagner \\ Massachusetts Institute of Technology and MGH-NMR Center
}

Anat Maril and Daniel L. Schacter

Harvard University

\begin{abstract}
Human memory consists of multiple forms, including priming and explicit memory. Although considerable evidence indicates that priming and explicit memory are functionally and neuroanatomically distinct, little is know about when and how these different forms of memory interact. Here, behavioral and functional magnetic resonance imaging (fMRI) methods were used to examine a novel and counterintuitive hypothesis: Priming during episodic encoding may be negatively associated with subsequent explicit memory. Using an experimental design that exploited known properties of spacing or lag effects, the magnitudes of behavioral and neural priming during a second study episode were varied and the relation
\end{abstract}

between these magnitudes of priming during re-encoding and performance on a subsequent explicit memory test was examined. Results revealed that greater behavioral priming (reduced reaction times) and neural priming (reduced left inferior prefrontal brain activation) during re-encoding were associated with lower levels of subsequent explicit memory. Moreover, those subjects who demonstrated greater behavioral and neural priming effects during re-encoding following a long lag tended to demonstrate the least benefit in subsequent explicit memory due to this second study episode. These findings suggest that priming for past experiences can hinder new episodic encoding.

\section{INTRODUCTION}

Extensive behavioral and neuropsychological evidence demonstrates that human long-term memory is not a unitary faculty, but rather consists of multiple forms of learning that differ in their processing characteristics and underlying neural substrates (Schacter \& Tulving, 1994). Neuropsychological studies have revealed that medialtemporal lobe regions are critical for episodic memorya form of explicit memory that supports the ability to consciously remember a past experience. In contrast, repetition priming - a non-conscious facilitation or biasing of stimulus processing that derives from implicit memory for past stimulus processing - is relatively preserved following medial-temporal lobe insult (Gabrieli, 1998; Cohen \& Eichenbaum, 1993; Schacter, Chiu, \& Ochsner, 1993; Squire, 1992).

Dissociations between explicit and implicit forms of memory have played a preeminent role in contemporary theorizing about memory (Schacter \& Tulving, 1994). Here, we examined a fundamental question regarding the relation between these two forms of memory that has thus far been largely overlooked: When and how do these different forms of memory interact? Combining behavioral and functional magnetic resonance imaging (fMRI) methods, we explored the possible "cross-talk"

between priming, a form of implicit memory, and episodic encoding, a form of explicit memory.

Episodic encoding refers to the processes that transform incoming information into an enduring memory representation that supports later conscious remembering (Tulving, 1983). Positron emission tomography (PET) and fMRI studies concerned with episodic encoding have shown activations in a variety of brain regions under conditions in which participants intentionally or incidentally encode information (Wagner, Koutstaal, \& Schacter, 1999; Buckner \& Koutstaal, 1998; Nyberg, 1998; Nyberg, Cabeza, \& Tulving, 1996). In addition to encoding-related activation in medial-temporal lobe regions (Schacter \& Wagner, 1999; Lepage, Habib, \& Tulving, 1998) that would be predicted from the neuropsychological literature, neuroimaging studies have consistently revealed greater activation in inferior prefrontal cortices during encoding conditions associated with superior long-term retention. Although the specific inferior prefrontal regions that support episodic encoding depend on the nature of the to-be-encoded material and on the processes engaged during encoding (Wagner, 1999; Kelley et al., 1998; Klingberg \& Roland, 1998; Wagner, Poldrack, Desmond, Glover, \& Gabrieli, 1998a), activation in left inferior prefrontal cortex (LIPC) 
is observed reliably during verbal episodic encoding (Gabrieli et al., 1996; Fletcher et al., 1995; Kapur et al., 1994).

Recent evidence indicates that, all else being equal, subsequent memory will be superior when there is greater recruitment of anterior- and posterior-LIPC regions during the encoding of a verbal experience (Kirchhoff, Wagner, Maril, \& Stern, 2000; Henson, Rugg, Shallice, Josephs, \& Dolan, 1999; Wagner et al., 1998b). Specifically, the encoding of words that were subsequently remembered was associated with greater LIPC activation relative to the encoding of words that were subsequently forgotten. One important implication of this finding is that factors that decrease the magnitude of LIPC activation during an experience should have negative consequences for episodic encoding-and subsequent explicit retrieval-of that experience.

Neuroimaging studies of repetition priming indicate that one factor influencing the magnitude of LIPC activation during the encoding of a stimulus is whether the stimulus has been previously processed. In particular, shorter reaction times (RTs), along with decreased anterior- and posterior-LIPC activation, have been observed during repeated relative to the initial semantic processing of a verbal stimulus (Schacter \& Buckner, 1998; Demb et al., 1995; Raichle et al., 1994). Although limited data are currently available about the status of LIPC priming effects following medial-temporal lobe insult, initial results indicate that similar repetitioninduced reductions in LIPC activation and in RTs are observed in individuals with global amnesia (Buckner \& Koutstaal, 1998; Gabrieli, Poldrack, \& Desmond, 1998). Thus, these behavioral and neural priming effects likely reflect the facilitative consequences of implicit memory for the initial processing experience, perhaps reflecting a computational benefit during repeated processing.

Evidence that the magnitude of LIPC activation during encoding is predictive of later explicit memory, and that priming results in reduced LIPC activation during re-encoding of a stimulus, raises an intriguing but previously unexamined possibility: Priming for past experiences can hinder new episodic encoding. According to this counterintuitive hypothesis, greater magnitudes of priming during the re-encoding of an item will be associated with lower levels of subsequent explicit memory for that item. To test this hypothesis, we varied the temporal lag between the initial and repeated encoding episodes with a word (2 min in the "Short-Lag" condition and $25 \mathrm{hr}$ in the "Long-Lag" condition). We predicted greater behavioral and neural priming during re-encoding following a shorter relative to a longer lag between the two encoding episodes. Previous studies of the lag or spacing effect have shown that repetitions of an item after short lags produce lower levels of subsequent explicit memory than do repetitions of the item after long lags (Greene, 1992; Melton, 1967; Ebbinghaus, 1885/1964). Thus, when explicit memory was examined $48 \mathrm{hr}$ later, we predicted worse long-term retention in the condition expected to be associated with greater behavioral and neural priming (i.e., the ShortLag condition). Moreover, we anticipated that, even when holding lag constant, greater magnitudes of behavioral and neural priming during re-encoding would be associated with worse subsequent explicit memory.

The experiment consisted of three sessions, distributed across a 4-day window (Figure 1). On Day 1, participants incidentally encoded a list of words, making an abstract/concrete decision for each word. fMRI scanning was not conducted during this encoding phase, and participants returned home following completion of the list. Approximately $25 \mathrm{hr}$ later (Day 2), seven study-scan sets were conducted. Immediately prior to each fMRI scan, participants incidentally encoded a list of previously unstudied words. Following presentation of the study list, fMRI scanning was performed while participants incidentally encoded a list of words that contained three types of word trials: reencoding of Day 1 words (Long-Lag trials), re-encoding of the immediately preceding Day 2 words (Short-Lag trials), and initial encoding of previously unstudied
Figure 1. Schematic diagram of the experimental procedure. (a) Initial, non-scanned encoding of Long-Lag words occurred on Day 1. (b) Initial, nonscanned encoding of Short-Lag words occurred on Day 2, followed by fMRI scanning (indicated by dashed lines) during re-encoding of the Long- and Short-Lag words as well as initial encoding of the Oncepresented items. There were seven study-scan sets on Day 2. (c) Explicit memory was assessed $48 \mathrm{hr}$ after scanning.

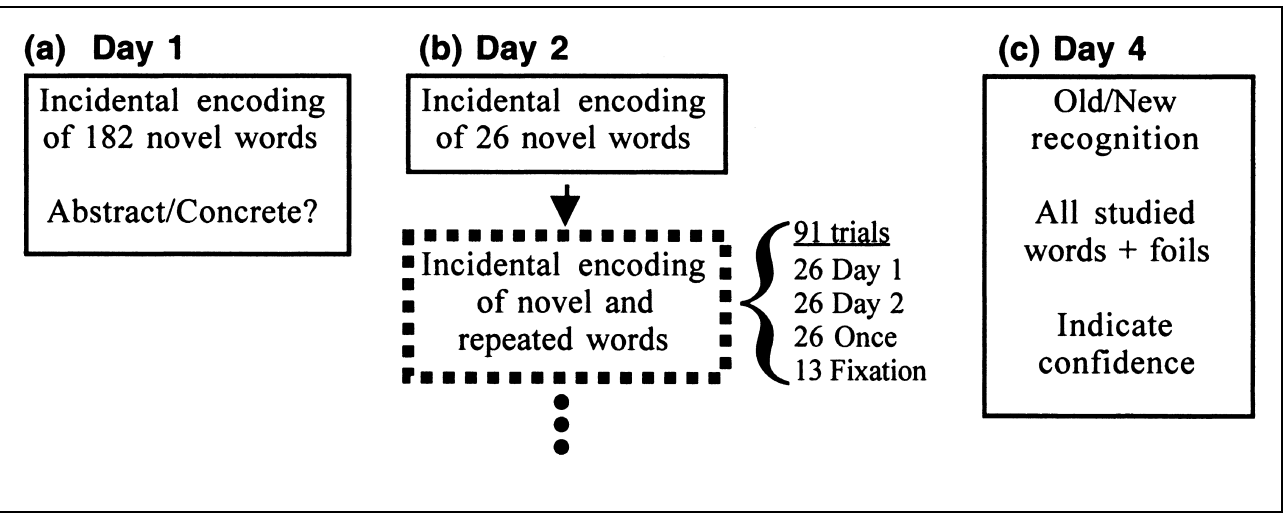


words (Once-presented trials). The Once-presented trials served as the baseline for computation of behavioral and neural priming in the Long-Lag and ShortLag conditions. Upon completion of the study-scan sets, participants again returned home. Approximately $48 \mathrm{hr}$ later (Day 4), long-term retention for all studied words (Long-Lag, Short-Lag, and Once-presented) was assessed using an explicit old/new recognition memory test that also included confidence judgments ("high" or "low" confidence).

\section{RESULTS}

\section{Behavioral Measures of Priming}

Mean median RTs to make the abstract/concrete decision during scanning on Day 2 declined from Oncepresented (777 msec) to Long-Lag (763 $\mathrm{msec}$ ) to Short-Lag $(720 \mathrm{msec})$ trials $(F(2,22)=26.55, p<$ .0001). Importantly, the RTs during re-encoding following a Short-Lag were significantly faster than those following a Long-Lag $(F(1,11)=27.27, p<.0001)$ and during initial encoding of the Once-presented words $(F(1,11)=49.18, p<.0001)$. Thus, behavioral priming was greater following a shorter than following a longer lag between encoding episodes. There was a trend for behavioral priming in the Long-Lag condition $(F(1,11)=3.21, p<.09)$.

\section{fMRI Measures of Priming}

The functional neuroimaging data revealed that numerous brain regions were engaged when comparing initial episodic encoding (Once-presented words) to the lower-level Fixation baseline, including regions in the LIPC and the left inferior temporal cortex (Figure $2 a$ ). However, only a subset of these regions demonstrated a significant repetition priming effect, i.e., greater activation during initial episodic encoding (Once-presented) relative to repeated episodic encoding (collapsed across Long-Lag and Short-Lag). Repetition priming effects were predominantly situated in the LIPC and the left inferior temporal regions (Figure $2 \mathrm{~b})$, including anterior-LIPC ( $\sim$ Brodmann's area [BA] $45 / 47)$, posterior-LIPC ( $\sim \mathrm{BA} 44 / 6)$, and left fusiform cortex $(\sim \mathrm{BA} 37)$.

Importantly, as with the measures of behavioral priming, a direct voxel-based comparison of the Long-Lag and Short-Lag trials revealed greater neural priming during re-encoding following a shorter than following a longer lag, with these effects being restricted to the LIPC and the left fusiform regions (Figure 3). Region-of-interest (ROI) analyses that examined the influence of lag duration on the magnitude of the LIPC and left fusiform priming effects observed when comparing Once-presented to all repeated trials revealed graded priming across lag (peak coordinates can be found in Figure 2). Specifically, for all ROIs, there was a significant trial type $\times$ time-point interaction (all $\left.F_{S}(14,154)>3.92, p<.0005\right)$. Planned contrasts revealed that, in all ROIs, there was significant neural priming in the Short-Lag condition (i.e., Once-presented $>$ Short-Lag $($ all $F s(1,11)>14.46, p<$ $.002)$. Moreover, with the exception of one left fusiform region $(-43,-58,-9 ; F(1,11)>2.20, p<.15)$, there also was significant neural priming in the LongLag condition (i.e., Once-presented > Long-Lag (all $F s(1,11)>5.75, p<.05)$. Critically, in all ROIs, there was greater priming in the Short-Lag relative to the

Figure 2. Neural correlates of episodic encoding and repetition priming. (a) Group activation maps are overlaid on averaged anatomic sections through peak activations in left prefrontal and temporal regions demonstrating greater activation during Once-presented relative to Fixation trials. Regions associated with the initial encoding of Once-presented trials included the anterior-LIPC (first and second columns), posterior-LIPC (third column), and left fusiform and lingual gyri (fourth column; indicated by red arrow). Extent rostral to the

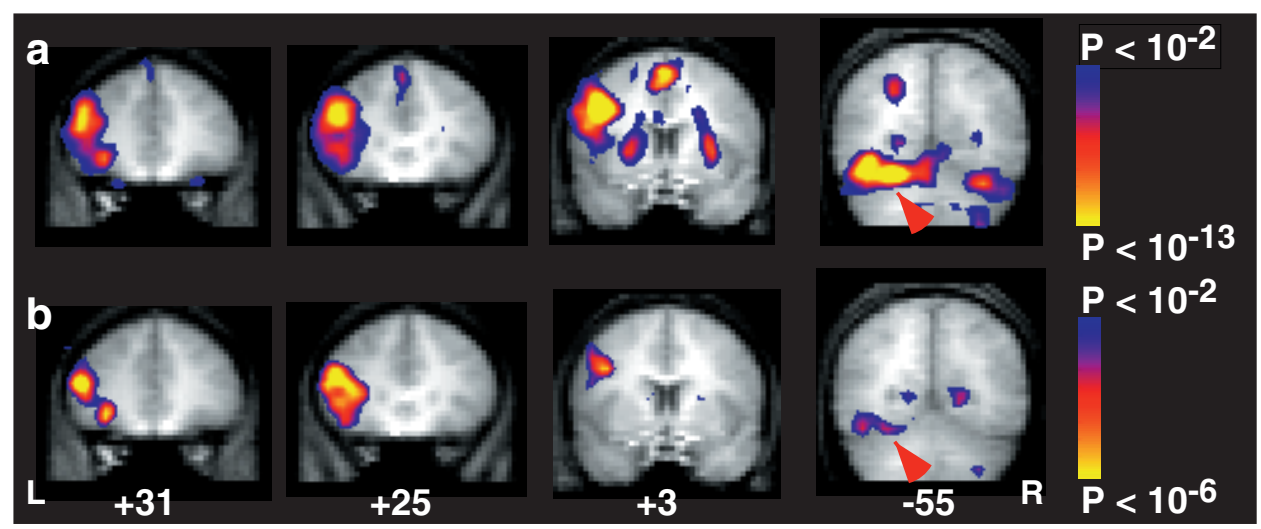
anterior commissure is indicated below each section in millimeters. (b) Left frontal and temporal regions also displayed significant priming effects, demonstrating greater activation during Once-presented trials than during re-encoding trials. Priming effects were observed in the anterior-LIPC ( $\sim \mathrm{BA} 45 / 47:-50,19,9$ and $-46,31,15 ; \sim \mathrm{BA} 47:-31,31,-3 ; \sim \mathrm{BA} 45 / 9:-46,22,21)$, posterior-LIPC/precentral $(\sim \mathrm{BA} 44 / 6:-40,3,31 ; \sim \mathrm{BA} 6:-46,0$, $43)$, left frontal operculum ( $\sim \mathrm{BA} 45 / 47:-37,25,15)$, and left fusiform/inferior temporal cortex ( BA 37: $-31,-55,-12 ;-25,-52,-12$; and -43 , $-58,-9)$. Other regions demonstrating priming effects were bilateral-posterior cingulate/lingual cortex ( BA 30/29/19: 15, $-49,6$ and $-12,-49,3$; see fourth column), and (not shown) medial cuneus/lingual ( $\sim$ BA 17: 0, $-77,12)$, medial frontal $(\sim \mathrm{BA} 6 / 8:-3,13,50)$, and left inferior occipital $(\sim$ BA 18: $-28,-87,0)$ cortices. 
Figure 3. Activation maps encompassing frontal and temporal regions that demonstrated less activation during the re-encoding of words following a Short-Lag relative to following a Long-Lag (i.e., greater neural priming in the Short-Lag condition). Displayed at the left are activation foci demonstrating less activation following a Short-Lag relative to a Long-Lag: (a) posteriorLIPC, bordering precentral gyrus ( $\sim \mathrm{BA} 44 / 6:-40,6,28$; $\sim$ BA 44/9: $-50,16,31$; $\sim$ BA 6 : $-40,0,40)$, (b) anterior-LIPC ( BA 45/47: $-46,28,15 ;-46$, 28,3 ; and $-53,19,6$ ), and (c) left fusiform/inferior temporal cortices ( BA 37: $-46,-49$, $-15 ;-46,-68,3$; and -50 , $-58,-12)$. Differential priming was also observed in left frontal operculum ( $\sim \mathrm{BA} 45 / 47:-31$, $25,12)$, left middle frontal $(\sim \mathrm{BA}$ 6: $-43,3,53)$, and medial frontal ( BA 6: $-3,9,50)$ regions (not shown). At the right are averaged event-related time courses from posteriorLIPC, anterior-LIPC, and left fusiform regions that demonstrated a priming effect when comparing Once-presented trials to all re-encoding trials Greater activation was observed during the Once-presented relative to the Long-Lag trials, and during the Long-Lag trials relative to the Short-Lag trials.
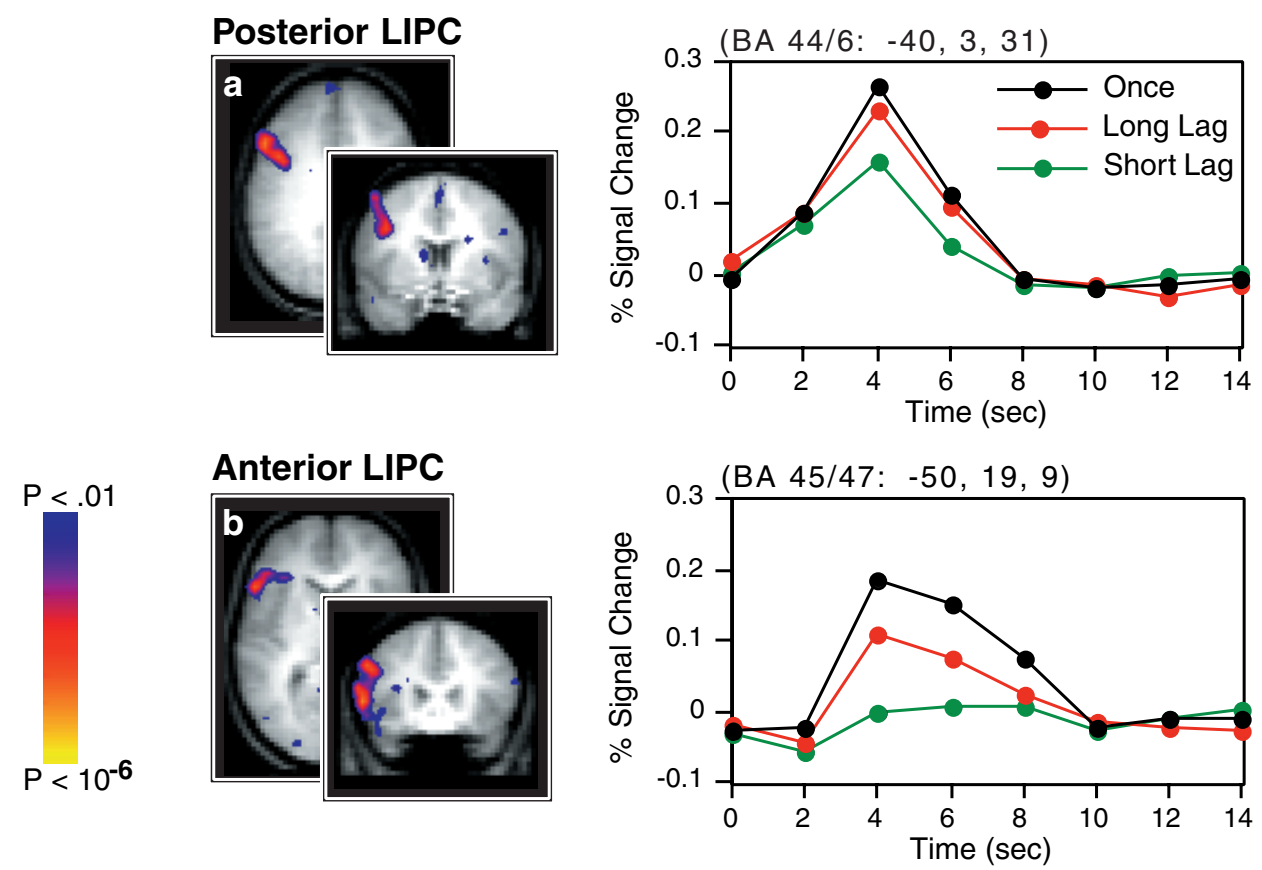

Fusiform Gyrus
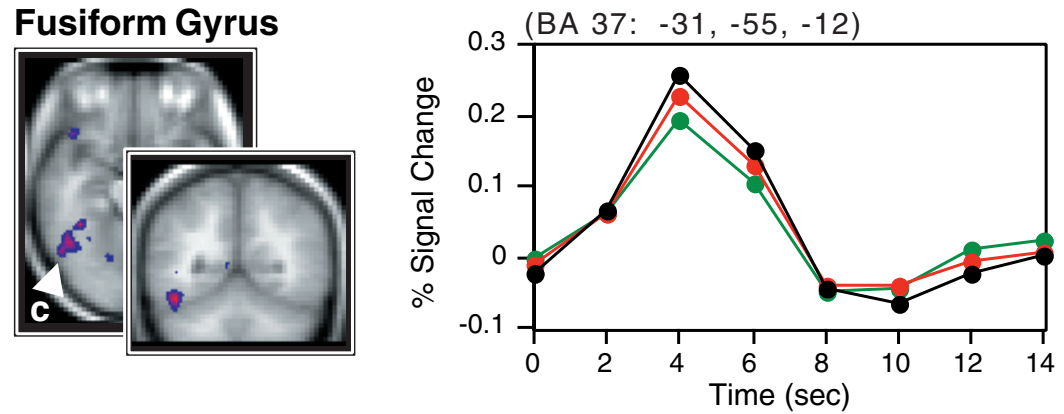

Long-Lag condition (i.e., Long-Lag $>$ Short-Lag (all $\left.F_{\mathrm{S}}(1,11)>5.37, p<.05\right)$. Thus, LIPC and left fusiform activation declined from initial encoding (Once-presented), to re-encoding following a Long-Lag, to reencoding following a Short-Lag (Figure 3).

\section{Association Between Explicit Memory and Priming}

We next examined a key prediction of our hypothesis: That the condition yielding greater priming during reencoding (Short-Lag) would be associated with lower levels of subsequent explicit memory compared to the condition yielding less priming (Long-Lag). Explicit memory scores (pHit-pFalse alarm) on the recognition memory test, computed both when collapsing across confidence levels ("high" and "low") and separately for "high-confidence" responses, differed across conditions (overall: $F(2,22)=85.12, p<.0001$; high confidence: $F(2,22)=72.15, p<$. 0001). Consistent with the predictions, overall subsequent explicit memory declined from the Long-Lag (35\%) to Short-Lag (31\%) condition $(F(1,11)=13.56, p<.005)$, and from the Short-Lag to Once-presented $(22 \%)$ condition $(F(1,11)$ $=81.00, p<.0001)$; the corresponding overall hit rates were 77,73 , and $64 \%$, respectively. Similarly, "highconfidence" subsequent explicit memory declined from Long-Lag (32\%) to Short Lag $(25 \%)(F(1,11)=31.23, p$ $<.0001)$, and from Short-Lag to Once-presented $(17 \%)(F(1,11)=41.14, p<.0001)$; the corresponding "high-confidence" hit rates were 46, 38, and 30\%, respectively. Thus, a negative association between magnitude of priming and subsequent explicit memory was observed.

To further explore the association between priming and explicit memory, the correlation between the magnitude of priming and subsequent "high-confidence" recognition was explored separately for the Long- and Short-Lag conditions. That is, lag was held constant and the relation between priming and subsequent explicit memory across-subjects was explored. For Long-Lag trials (Figure 4a), there was a negative correlation $(r$ $=-.79 ; t(10)=-4.07, p<.005)$ between the magni- 
Figure 4. Negative correlations between subsequent explicit memory and behavioral and neural measures of priming during re-encoding. (a) Acrosssubjects, there was a negative association between behavioral priming during the Long-Lag trials (RT difference, in milliseconds (msec), between Oncepresented and Long-Lag trials) and subsequent explicit memory ("high-confidence" recognition difference, in probability, between Long-Lag and Once-

presented trials). Across-subjects, in (b) the posterior-LIPC ( $\sim$ BA 44/6: $-40,3,31)$ and (c) the anterior-LIPC ( $\sim$ BA $45 / 47:-50,19$, 9), there were negative associations between neural priming during the Long-Lag trials (percent signal change difference between Once-presented and Long-Lag trials) and subsequent explicit memory. The negative correlation in the anterior-LIPC was reliable both when the leftmost outlying point was included, and when excluded from the analysis. These correlations indicate that those subjects demonstrating greater priming during re-encoding tended to benefit less from this second study episode, as indexed by recognition memory. tude of behavioral priming during re-encoding (RT difference between Once-presented and Long-Lag trials) and subsequent explicit memory ("high-confidence" recognition difference between Long-Lag and Oncepresented). Similarly, there was a negative correlation between the magnitude of neural priming (percent signal change difference between Once-presented and Long-Lag) in the LIPC and the left fusiform ROIs observed to demonstrate priming when comparing the Once-presented to all repetition trials (Figure $4 \mathrm{~b}$ and c) and subsequent explicit memory. Specifically, negative correlations were observed in anterior-LIPC $(-50,19,9: r=-.54, t(10)=-2.03, p<.05$; and $-31,31,-3: r=-.58, t(10)=-2.25, p<.05)$, posterior-LIPC/precentral $(-40,3,31: r=-.59, t(10)=$ $-2.31, p<.05)$, and left fusiform/inferior temporal cortex $(-31,-55,-12: r=-.53, t(10)=-1.87, p<$ .05 ; and $-25,-52,-12: r=-.50, t(10)=-1.83, p<$ $.05)$. For the Short-Lag trials, reliable correlations were not observed between explicit memory and either behavioral or neural measures of priming. These null effects may partially derive from a restricted range for the explicit memory measure for this condition; the range of this measure for the Short-Lag condition was only .05-.11 (excluding an outlier), whereas the range in the Long-Lag condition was .09-.26.

\section{DISCUSSION}

The present study demonstrates that behavioral and neural measures of priming during the reprocessing of a stimulus are negatively associated with subsequent memory for that stimulus, thus providing support for the hypothesis that priming from past experiences impairs new episodic encoding. The encoding condition associated with greater behavioral and neural priming during re-encoding (Short-Lag) was also associated with worse subsequent explicit memory. Moreover, within the Long-Lag condition, behavioral and neural measures of priming were negatively associated with subsequent memory. These data suggest that there is "cross-talk" between implicit and explicit forms of memory, with implicit memory for the past hindering new episodic learning (note, however, that causality cannot be inferred from correlational data).

The observation that behavioral priming and neural priming on the abstract/concrete decision task are negatively correlated with subsequent explicit memory within the Long-Lag condition provides further evidence that these priming effects derive from implicit memory. Specifically, if the reductions in RTs and in LIPC activation during the second study episode were due to successful explicit retrieval of the initial study episode-rather than due to implicit memory-then the observed negative correlations would suggest that those subjects demonstrating better successful explicit retrieval during the second study episode actually demonstrated worse memory performance on the final recognition memory test. Such an outcome would be surprising as one might expect that those subjects demonstrating better explicit memory during the second study episode would also demonstrate better explicit memory on the final test. Moreover, there is a considerable behavioral literature demonstrating that retrieval practice has a robust facilitative effect on later explicit memory (Bjork, 1975). Thus, if explicit retrieval were the source of the priming effects during the second study episode, then more successful retrieval during this second study should have been associated with a greater benefit to performance on the final memory test. However, the opposite pattern was observed in this study. These data, in conjunction with earlier observations that amnesic patients demonstrate intact behavioral and neural priming on the abstract/ concrete decision task (Buckner \& Koutstaal, 1998; 
Gabrieli et al., 1998), indicate that the LIPC and behavioral priming effects derive from implicit memory. Critically, the observed negative correlations between priming and subsequent explicit memory provide the first evidence, to our knowledge, suggesting that such priming can have negative consequences for explicit learning.

Although the current study does not directly implicate a specific underlying mechanism through which priming and explicit memory interact, we posit one candidate: Priming may impair new episodic encoding and subsequent explicit memory by reducing encoding variability. Behavioral studies have demonstrated that encoding variability enhances subsequent explicit memory because it results in the encoding of multiple event features or attributes, thus providing multiple retrieval routes to a particular episodic memory (Martin, 1968). Priming may reduce encoding variability by increasing the probability that the same task-relevant stimulus features will be processed during a subsequent encoding event as were processed during an initial encoding event.

From this perspective, activation in the inferior prefrontal cortex during episodic encoding is thought to reflect directed attention to task-relevant stimulus features (e.g., those semantic features that allow for classification of a word as abstract or concrete) (Thompson-Schill, D'Esposito, Aguirre, \& Farah, 1997; Wagner, Desmond, Demb, Glover, \& Gabrieli, 1997). This allocation of attention to task-relevant features may result in priming of these features during a subsequent processing experience. In addition, this attentional allocation may render the features available for input to the medial-temporal memory system that binds features into an explicit or declarative memory trace (Moscovitch, 1992). During re-encoding, priming for the initial processing may serve to make these taskor goal-relevant features more readily available than task-irrelevant features. This greater accessibility of task-relevant features would reduce the demand on prefrontally mediated attentional operations, thus resulting in decreased LIPC activation (Thompson-Schill, D'Esposito, \& Kan, 1999). Moreover, the greater accessibility of these features would decrease the likelihood that other features-be they task-relevant or task-irrelevant-would be attended (Gartman \& Johnson, 1992, Jacoby \& Craik, 1979). In this manner, priming serves to bias re-encoding and to reduce encoding variability, because the same task-relevant features would serve as input to the medial-temporal memory system. From this perspective, priming tends to yield a stereotyped or sparse re-encoding experience (Wiggs \& Martin, 1998). However, as priming declines, the probability of attending to other task-relevant or task-irrelevant features during re-encoding would increase, thus increasing encoding variability and, ultimately, the efficacy of the encoding experience.
The current observations provide evidence for a novel cognitive and neurobiological interpretation of the spacing or lag effect, first described by Ebbinghaus over 100 years ago (Ebbinghaus, 1885/1964). At the cognitive level, the present results suggest that the lag effect may, at least partially, derive from attenuation of the negative consequences of priming that occurs with a longer lag (Challis \& Sidhu, 1993). At the neurobiological level, these results indicate that the lag effect is associated with differential engagement of inferior prefrontal regions that are thought to play a critical role in episodic encoding. We anticipate that these novel interpretations represent some of the first of many insights that will be derived from consideration of the interactions between forms of memory.

\section{METHODS}

\section{Participants}

All participants were right-handed, native speakers of English (five men, seven women; ages 18-27 years), with normal or corrected-to-normal vision, and received US $\$ 50$ for participation. Informed consent was obtained in a manner approved by the Human Studies Committee of the Massachusetts General Hospital.

\section{Stimuli and Behavioral Procedures}

Stimuli consisted of abstract (e.g., LOVE) and concrete (e.g., CHAIR) words printed in uppercase letters; stimuli were counterbalanced across conditions. A schematic of the procedure is illustrated in Figure 1. (a) On Day 1, participants incidentally encoded 182 words (i.e., there was no mention that explicit memory would be probed on Day 4). Words were presented individually, one word every $2 \mathrm{sec}$ (1-sec stimulus duration, followed by 1 -sec visual fixation on a "+" sign). To minimize fatigue, 15 -sec breaks were provided after each seventh of the list. These words constituted the "Long-Lag" items. (b) On Day 2, seven study-scan sets were presented. Prior to each scan, 26 previously unstudied words were encoded; these words constituted the "Short-Lag" items. A 91-trial fMRI scan followed this study list. Trials consisted of 26 words previously encoded on Day 1 (Long-Lag), 26 words previously encoded immediately prior to the scan (Short-Lag), 26 words not previously studied (Once-presented), and 13 fixation trials (fixation for the entire trial). Trial types were rapidly intermixed with each trial lasting $2 \mathrm{sec}$. (c) An old/new recognition test was administered following a 48-hr retention interval. All previously studied words and a set of unstudied foils were presented during the recognition test. Words were presented individually with self-paced timing. Participants indicated whether they thought the item was studied, and reported their confidence ("high" or "low") when responding "studied." To 
minimize fatigue, 20 -sec breaks were provided after every seventh of the test. For all phases of the experiment, responses were indicated via left-handed key presses.

\section{fMRI Procedures}

Echo-planar and conventional imaging was performed on a 3.0-T GE Signa scanner with an ANMR upgrade. Imaging procedures were identical to those previously reported (Wagner et al., 1998b). Conventional structural images included a high-resolution rf-spoiled GRASS sequence (SPGR; 60 slice sagittal, 2.8-mm thickness), and a set of $T_{1}$ flow-weighted anatomic images coplanar with the functional echo-planar images (16 slices, aligned with the plane intersecting the anterior and posterior commissures, $0.78-\mathrm{mm}$ in-plane resolution, 7$\mathrm{mm}$ thickness, skip 1-mm between slices). The flowweighted images served as an intermediate to align the echo-planar images to the SPGR images. Echo-planar imaging consisted of an automated shim procedure to improve $B_{0}$ magnetic field homogeneity, and $T_{2}{ }^{*}$ weighted gradient-echo functional images (99 sequential whole-brain acquisitions, 16 slices, 3.125-mm in-plane resolution, 7-mm thickness, skip $1 \mathrm{~mm}$; TR $=2 \mathrm{sec}$; TE $=30$ msec; flip angle $=90^{\circ}$ ). A General Electric quadrature head coil was used for signal reception. The subject's head was immobilized with pillows, cushions, and a restraining strap to reduce motion artifact.

The procedures for selective averaging and statistical map generation for rapidly intermixed event-related trials have been described elsewhere (Buckner et al., 1998; Dale \& Buckner, 1997). Briefly, data from individual functional runs were normalized to correct for signal intensity changes and temporal drift. Normalized data were then selectively averaged in relation to the beginning of each trial type both within-subjects and across-subjects (much like event-related potential [ERP] data are averaged). Finally, statistical activation maps were constructed based on the averaged event-related responses for each trial type.

Normalization of each functional run involved: (a) scaling of whole-brain signal intensity to a fixed value of 1000, (b) linear slope removal on a voxel-by-voxel basis to counteract effects of drift (Bandettini, Jesmanowicz, Wong, \& Hyde, 1993), (c) spatial filtering with a 1.5-voxel radius Hanning filter, and (d) removal of the mean signal intensity on a voxel-by-voxel basis. Normalized functional runs were selectively averaged within subject, resulting in eight mean images (16 sec at TR $=2 \mathrm{sec}$ ) for each trial type as well as the variance for each of the eight images per trial type (Buckner et al., 1998; Dale \& Buckner, 1997). These mean and variance images were subsequently transformed into Talairach and Tournoux (1988) atlas space. Across-subject data averaging consisted of weighting the means and variance by the number of trials contributed by each subject. With respect to the present design, this procedure is equivalent to weighting the subjects equally, because all subjects contributed an equal number of trials per condition. The SPGR anatomical images were averaged across-subjects to yield a mean anatomy image onto which the functional data were projected.

Activation maps were constructed using a $t$ statistic (Buckner et al., 1998). For this analysis, a set of predicted hemodynamic response functions was generated with the onset delay of the hemodynamic response varied over time; the delay varied in 1-sec steps from -1.0 to $5.0 \mathrm{sec}$ with respect to the trial onset. Gamma functions were used as the base shape with fixed parameters $\delta=2.5 \mathrm{sec}$ and $\tau=1.25 \mathrm{sec}$ ( Buckner et al., 1998; Dale \& Buckner, 1997) and a delay parameter. Varying the onset delay of the predicted hemodynamic curve can be critical for detection of activation changes throughout cortical and subcortical structures, as regional variability in the timing onset of the hemodynamic response has been observed (e.g., Schacter, Bucker, Koutstaal, Dale, \& Rosen, 1997). However, in this study, the onset delay of the predicted curve that resulted in peak foci of activation ranged from a lag of $0-2 \mathrm{sec}$ (or one TR), perhaps partially reflecting variability due to slice timing acquisition (for which our procedures did not adjust).

Statistical maps were generated by calculating the covariance between the observed signal difference between trial types and the predicted hemodynamic response functions, and represent the maximal $t$ obtained. Fixation trial events were subtracted from the Oncepresented word trials to identify regions engaged during initial episodic encoding. Re-encoding trials (collapsed across Long-Lag and Short-Lag trials) were subtracted from the Once-presented trials to identify regions demonstrating a neural priming effect. To examine differential priming across lag, Long-Lag trials were subtracted from Short-Lag trials. Clusters of five or more voxels exceeding a statistical threshold of $p<0.001$ were considered significant foci of activation.

To further explore the effect of lag on priming, ROI analyses were performed for all left frontal and temporal regions observed when comparing the Once-presented trials to all re-encoding trials. Time courses were derived from a 3-D region surrounding the peak voxel, and reflect percent signal change from Fixation. Regions were defined using an automated algorithm that identified all contiguous voxels within $10 \mathrm{~mm}$ of the peak that reached significance. Mixed-effect analyses of variance were performed, treating percent signal change from Fixation as the dependent variable and trial type (Oncepresented, Long-Lag, Short-Lag) and time-point (0-14 sec from stimulus onset) as repeated factors; subjects were treated as a random effect. The effect of trial type was further explored in regions demonstrating a trial type $\times$ time-point interaction through planned contrasts comparing the percent signal change for each of the 
three word-trial types at the time-point corresponding to the peak (highest amplitude) response in the ROI when defined using the mean of all three word-trial types. A Huynh-Feldt correction for non-sphericity was implemented for these ROI analyses, as well as for analyses of the behavioral data.

\section{Acknowledgments}

Supported by the National Institute of Mental Health (MH60941), National Institute on Aging (AG05778), the Surdna Foundation, and P. E. Newton. We thank W. Koutstaal for insightful comments and discussion, and A. Dale for technical assistance.

The data reported in this experiment have been deposited in National fMRI Data Center (http://www.fmridc.org). The accession number is 2-2000-11142.

Reprint requests should be sent to Anthony D. Wagner, Department of Brain and Cognitive Science, NE20-436, MIT, Cambridge, MA 02139.

\section{REFERENCES}

Bandettini. P. A.. Jesmanowicz, A., Wong. E. C.. \& Hvde. I. S. (1993). Processing strategies for time-course data sets in functional MRI of the human brain. Magnetic Resonance in Medicine. 30. 161-173.

Bjork, R. A. (1975). Retrieval as a memory modifier. In R. Solso (Ed.), Information processing and cognition: The Loyola Symposium (pp. 123-144). Hillsdale, NJ: Erlbaum.

Buckner. R. L. Goodman. I. Burock. M. Rotte. M. Koutstaal. W. Schacter, D. Rosen, B. \& Dale, A. M. (1998). Functionalanatomic correlates of obiect priming in humans revealed bv rapid presentation event-related fMRI. Neuron, 20, 285-226.

Buckner, R. L., \& Koutstaal, W. (1998). Functional neuroimaging studies of encoding, priming, and explicit memory retrieval. Proceedings of the National Academy of Science, U.S.A. 95, 891-898.

Challis, B. H., \& Sidhu, R. (1993). Dissociative effect of massed repetition on implicit and explicit measures of memory Lournal of Experimental Psychology: Learning. Memorv. and Cognition. 19. 115-127.

Cohen, N. J., \& Eichenbaum, H. (1993). Memory, amnesia, and the hippocampal system. Cambridge: MIT Press.

Dale. A. M. \& Buckner, R. L. (1997). Selective averaging of rapidly presented individual trials using fMRI. Human Brain Mapping. 5. 329-340.

Demb, J. B., Desmond, J. E., Wagner, A. D., Vaidya, C. J., Glover, G. H., \& Gabrieli, J. D. E. (1995). Semantic encoding and retrieval in the left inferior prefrontal cortex: A functional MRI study of task difficulty and process specificity. Journal of Neuroscience, 15, 5870-5878.

Ebbinghaus, H. (1885/1964). Memory: A contribution to experimental psychology. New York: Dover.

Fletcher, P. C. Frith. C. D.. Grasby, P. M., Shallice, T. Frackowiak, R. S. \& Dolan. R. J. (1995). Brain systems for encoding and retrieval of auditory-verbal memorv: An in vivo studv in humans. Brain. 118. 401-416.

Gabrieli. I. D. (1998). Cognitive neuroscience of human memorv. Annual Review of Psvchologv. 49. 87-115.

Gabrieli. J. D. E.. Desmond, I. E.. Demb. I. B., Wagner, A. D. Stone, M. V. Vaidva, C. I. \& Glover, G. H. (1926). Functional magnetic resonance imaging of semantic memorv processes in the frontal lobes. Psychological Science. 7. 278-283.
Gabrieli, J. D., Poldrack, R. A., \& Desmond, J. E. (1998). The role of left prefrontal cortex in language and memory. Proceedings of the National Academy of Science, U.S.A. 95, 906-913.

Gartman, L. M.. \& Johnson, N. F. (1972). Massed versus distributed repetition of homographs: A test of the differentialencoding hypothesis. Iournal of Verbal Learning \& Verbal Behavior. 11. 801-805.

Greene, R. L. (1992). Human memory: Paradigms and paradoxes. Hillsdale, NJ: Erlbaum.

Henson, R. N. A., Rugg, M. D., Shallice, T., Josephs, O., \& Dolan, R. J. (1999). Recollection and familiarity in recognition memory: An event-related functional magnetic resonance imaging study. Journal of Neuroscience, 19, 3962-3972.

Jacoby, L. L., \& Craik, F. I. M. (1979). In L. S. Cermak \& F. I. M. Craik (Eds.), Levels of processing in human memory. New York: Wiley.

Kapur, S., Craik, F. I. M., Tulving, E., Wilson, A. A., Houle, S., \& Brown, G. M. (1994). Neuroanatomical correlates of encoding in episodic memory: Levels of processing effect. Proceedings of the National Academy of Science, U.S.A. 91, 2008-2011.

Kellev, W. M., Miezin, F. M., McDermott, K. B., Buckner, R. L. Raichle. M. E. Cohen. N. I. Ollinger. I. M. Akbudak. E. Conturo, T. E. Snvder, A. Z. \& Petersen, S. E. (1998). Hemispheric specialization in human dorsal frontal cortex and medial temporal lobe for verbal and nonverbal memory encoding. Neuron, 20, 927-936.

Kirchhoff, B. A., Wagner, A. D., Maril, A., \& Stern, C. E. (2000). Prefrontal-temporal circuitry for episodic encoding and subsequent memory. Journal of Neuroscience, 20, 6173-6180.

Klingberg. T. \& Roland, P. E. (1998). Right prefrontal activation during encoding, but not during retrieval, in a non-verbal paired-associates task. Cerebral Cortex, 8, 73-79.

Lepage. M. Habib. R. \& Tulving. E. (1998). Hippocampal PET activations of memory encoding and retrieval: The HIPER model. Hippocampus, 8, 313-322.

Martin, E. (1968). Stimulus meaningfulness and pairedassociate transfer: An encoding variability hypothesis. Psvchological Review. 75. 421-441.

Melton, A. W. (1967). Repetition and retrieval from memory. Science. 158. 532.

Moscovitch. M. (1992). Memorv and working-with-memorv: A component process model based on modules and central svstems. Iournal of Cognitive Neuroscience. 4. $257-267$.

Nvberg. L. (1998). Mapping episodic memory. Behavioral Brain Research, 20, 107-114

Nyberg, L., Cabeza, R., \& Tulving, E. (1996). PET studies of encoding and retrieval: The HERA model. Psychonomic Bulletin and Review, 3, 135-148.

Raichle. M. E. Fiez. I. A. Videen. T. O.. Macleod. A. M. K. Pardo, I. V., Fox, P. T. \& Petersen, S. E. (1994). Practicerelated changes in human brain functional anatomv during nonmotor learning. Cerebral Cortex, 4, 8-26.

Schacter, D. L. \& Buckner, R. L. (1998). Priming and the brain. Neuron, 20. 185-195.

Schacter, D. L., Buckner, R. L. Koutstaal, W. Dale, A. M. \& Rosen, B. R. (1997). Late onset of anterior prefrontal activity during true and false recognition: An event-related fMRI studv. Neuroimage, 6. 259-269.

Schacter. D. L. Chiu. C. Y. \& Ochsner. K. N. (1993). Implicit memorv: A selective review. Annual Review of Neuroscience, 16, 159-182.

Schacter, D. L., \& Tulving, E. (Eds.) (1994). Memory systems 1994. Cambridge: MIT Press. 
Schacter. D. L. \& Wagner, A. D. (1999). Medial temporal lobe activations in fMRI and PET studies of episodic encoding and retrieval. Hitbocampus. 2. 7-24.

Squire, L. R. (1992). Memory and the hippocampus: A svnthesis from findings with rats, monkevs, and humans. Psvchological Review. 29. 195-231.

Talairach, J., \& Tournoux, P. (1988). A co-planar stereotactic atlas of the buman brain. New York: Thieme.

Thompson-Schill, S. L., D'Esposito, M., Aguirre, G. K., \& Farah, M. J. (1997). Role of left inferior prefrontal cortex in retrieval of semantic knowledge: A reevaluation. Proceedings of the National Academy of Science, U.S.A.. 94, 14792-14797.

Thompson-Schill, S. L.. D'Esposito, M.. \& Kan. I. P. (1999) Effects of repetition and competition on activity in left prefrontal cortex during word generation. Neuron. 23. 513-522.

Tulving, E. (1983). Elements of episodic memory. Cambridge: Cambridge University Press.

Wagner. A. D. (1999). Working memorv contributions to human learning and remembering. Neuron, 22, 19-22.

Wagner. A. D.. Desmond. I. E. Demb. I. B. Glover. G. H., \&
Gabrieli. I. D. E. (1997). Semantic repetition priming for verbal and pictorial knowledge: A functional MRI studv of left inferior prefrontal cortex. Iournal of Cognitive $\mathrm{Neu}$ roscience. 2. 714-726.

Wagner, A. D., Koutstaal, W., \& Schacter, D. L. (1999). When encoding yields remembering: Insights from event-related neuroimaging. Philosophical Transactions of the Royal Society of London, Series B: Biological Sciences. 354, 1283-1294.

Wagner, A. D., Poldrack, R. A., Desmond, J. E., Glover, G. H., \& Gabrieli, J. D. E. (1998a). Material-specific lateralization of prefrontal activation during episodic encoding and retrieval. NeuroReport, 9, 3711-3717.

Wagner, A. D. Schacter, D. L. Rotte, M. Koutstaal. W. . Maril. A. Dale. A. M. Rosen, B. R. \& Buckner. R. L. (1998b). Building memories: Remembering and forgetting of verbal experiences as predicted bv brain activity. Science. 281 1188-1191.

Wiggs, C. L. \& Martin. A. (1998). Properties and mechanisms of perceptual priming. Current Opinions in Neurobiologv. 8. $\underline{227-233}$ 


\section{This article has been cited by:}

1. E. A. Race, D. Badre, A. D. Wagner. 2009. Multiple Forms of Learning Yield Temporally Distinct Electrophysiological Repetition Effects. Cerebral Cortex . [CrossRef]

2. Elizabeth A. Race, Shanti Shanker, Anthony D. Wagner. 2009. Neural Priming in Human Frontal Cortex: Multiple Forms of Learning Reduce Demands on the Prefrontal Executive SystemNeural Priming in Human Frontal Cortex: Multiple Forms of Learning Reduce Demands on the Prefrontal Executive System. Journal of Cognitive Neuroscience 21:9, 1766-1781. [Abstract] [Full Text] [PDF] [PDF Plus]

3. Daniel E. Callan, Nicolas Schweighofer. 2009. Neural correlates of the spacing effect in explicit verbal semantic encoding support the deficient-processing theory. Human Brain Mapping NA-NA. [CrossRef]

4. Haline E. Schendan, Marta Kutas. 2007. Neurophysiological Evidence for the Time Course of Activation of Global Shape, Part, and Local Contour Representations during Visual Object Categorization and MemoryNeurophysiological Evidence for the Time Course of Activation of Global Shape, Part, and Local Contour Representations during Visual Object Categorization and Memory. Journal of Cognitive Neuroscience 19:5, 734-749. [Abstract] [PDF] [PDF Plus]

5. Thalia Wheatley, Jill Weisberg, Michael S. Beauchamp, Alex Martin . 2005. Automatic Priming of Semantically Related Words Reduces Activity in the Fusiform GyrusAutomatic Priming of Semantically Related Words Reduces Activity in the Fusiform Gyrus. Journal of Cognitive Neuroscience 17:12, 1871-1885. [Abstract] [PDF] [PDF Plus]

6. Gail O'Kane, Rachel Z. Insler, Anthony D. Wagner. 2005. Conceptual and perceptual novelty effects in human medial temporal cortex. Hippocampus 15:3, 326-332. [CrossRef]

7. Ian G. Dobbins , Jon S. Simons, Daniel L. Schacter . 2004. fMRI Evidence for Separable and Lateralized Prefrontal Memory Monitoring ProcessesfMRI Evidence for Separable and Lateralized Prefrontal Memory Monitoring Processes. Journal of Cognitive Neuroscience 16:6, 908-920. [Abstract] [PDF] [PDF Plus]

8. Chun-Siong Soon, Vinod Venkatraman, Michael W.L. Chee. 2003. Stimulus repetition and hemodynamic response refractoriness in event-related fMRI. Human Brain Mapping 20:1, 1-12. [CrossRef]

9. 2001. Current Awareness. NMR in Biomedicine 14:3, 217-222. [CrossRef] 